\title{
Photonic bandgaps in patterned waveguides of silicon-rich silicon dioxide
}

\author{
R. T. Neal, M. E. Zoorob, ${ }^{\text {a) }}$ M. D. Charlton, ${ }^{\text {a) }}$ and G. J. Parker ${ }^{a}$ \\ Department of Electronics \& Computer Science, University of Southampton, Southampton SO17 1BJ, \\ United Kingdom \\ C. E. Finlayson ${ }^{\text {b) }}$ and J. J. Baumberga) \\ Department of Physics and Astronomy, University of Southampton, Southampton SO17 1BJ, \\ United Kingdom
}

(Received 2 October 2003; accepted 5 February 2004)

\begin{abstract}
We describe waveguides of photoluminescent silicon-rich silicon dioxide, which have been patterned by triangular two-dimensional (2D) photonic crystals to give higher-order photonic bandgaps occurring within the luminescence band of the core material. Photonic crystal modification of the photoluminescence spectrum allows identification of angle-tuned photonic bandgaps, in close agreement with 2D plane wave expansion and finite-difference time domain simulations. We discuss the importance of these findings for the development of integrated optical circuitry based on silicon-compatible microelectronics. (C) 2004 American Institute of Physics.
\end{abstract}

[DOI: 10.1063/1.1690106]

Following the first demonstration of light emission from the silicon band edge, ${ }^{1}$ there has been intense interest in enhancing and controlling the emission of light from siliconbased nanostructures with a view to the development of inexpensive optoelectronic components. Methods have included anodic etching of silicon to create porous silicon, ${ }^{2}$ and the annealing of crystalline silicon/silicon dioxide superlattices. ${ }^{3}$ Such methods suffer from problems due to the complexity of the growth process or long-term instability of the material. We propose that luminescent nanocrystals formed in silicon-rich silicon dioxide (SRSO) waveguides through plasma-enhanced chemical vapor deposition $(\mathrm{PECVD})^{4}$ provides a more viable material platform, which is both compatible with complementary metal-oxidesemiconductor (CMOS) processes and stable enough for the development of luminescent silicon devices and highly engineered integrated optical circuits.

Photonic crystals (PCs) ${ }^{5,6}$ are periodic dielectric structures, which have the ability to restrict propagation of light into discrete energy bands and localize the optical fields to very small volumes of space. Consequently, PCs have important applications in controlling the flow of light in optoelectronic devices fabricated on a submicron scale, ${ }^{7-10}$ and PC waveguides promise many ways to miniaturize and integrate devices on high-functionality optical chips. PCs have recently been fabricated from luminescent materials such as GaAs, ${ }^{11}$ and porous silicon ${ }^{12}$ in an effort to enhance photoluminescence (PL) extraction efficiency.

Here, we fabricate and measure PC structures based on a two-dimensional (2D) triangular lattice of holes etched into waveguide layers based upon our previously demonstrated low-loss single-mode slab waveguides in SRSO by PECVD. ${ }^{13}$ The optical properties of the second- and thirdorder bandgaps result in modification of the luminescence

\footnotetext{
a) Also at: Mesophotonics Ltd, 2 Venture Rd, Chilworth Science Park, Southampton SO16 7NP, United Kingdom.

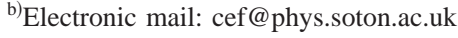

spectra observed experimentally. This is a demonstration of a PC bandgap effect in waveguides where the siliconcompatible core material is actively luminescent.

Lightly doped $p$-silicon wafers were thermally oxidized to a depth of $2.1 \mu \mathrm{m}$ and $450 \mathrm{~nm}$ of SRSO deposited upon the surface by PECVD. The percentage of silicon incorporated within the layer was controlled during deposition by varying the ratios of $\mathrm{SiH}_{4}$ and $\mathrm{N}_{2} \mathrm{O}$ while maintaining constant pressure. A 200-nm-thick cladding layer of $\mathrm{SiO}_{2}$ was deposited by PECVD, and the complete structure was annealed at $1150{ }^{\circ} \mathrm{C}$ for several hours to promote the formation of silicon nanocrystals. Resist was then spun onto the multilayer and patterned by direct write electron-beam lithography. Finally, a dry plasma etch $\left(\mathrm{CHF}_{3}+\mathrm{Ar}\right)$, transferred the pattern through the waveguide layers and part way through the $\mathrm{SiO}_{2}$ buffer layer to a depth of over $750 \mathrm{~nm}$.

Figure 1 shows the absorption of the unpatterned SRSO waveguide in contrast with the unmodified PL emerging normally from the layer. It can clearly be seen from the overlap between PL and absorption that light emitted from the SRSO suffers significant re-absorption at the shorter wavelength

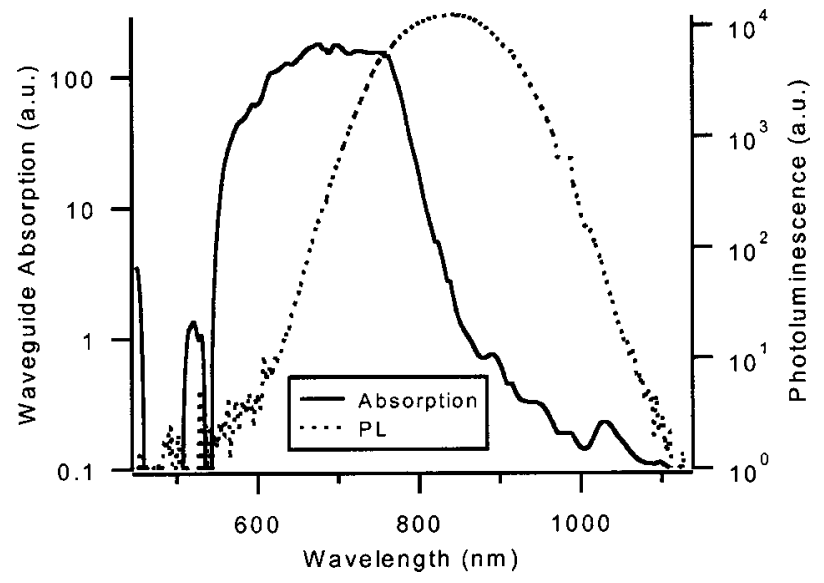

FIG. 1. Measured absorption (solid) and PL (dashed) spectra for SRSO. Excitation for the PL measurement was at a wavelength of $514 \mathrm{~nm}$. 


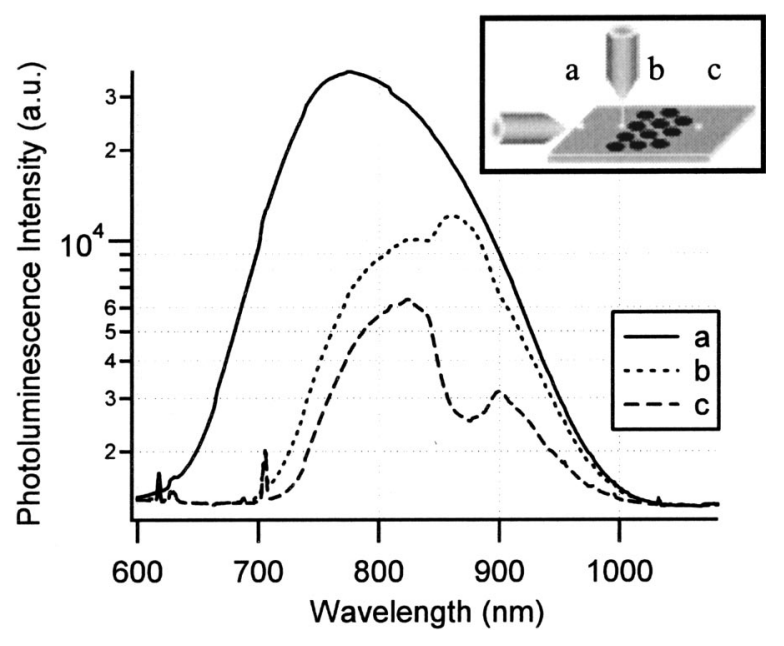

FIG. 2. PL emitted from the end facet of a PC-patterned SRSO waveguide. The inset shows schematically the location of the excitation spot for each curve. Light was collected at the waveguide facet (near spot "a") in each instance. Note that the background offset on these spectra is approximately 1100 counts.

end of the spectrum from the SRSO as it passes through the waveguide. The reduced transmission below $800 \mathrm{~nm}$ is caused by absorption from the Si nanocrystals in the silica matrix. The PL from the nanocrystals continues up to $\sim 1200$ $\mathrm{nm}$, but is not detected by the Si-CCD used here.

Measurements were carried out to investigate the luminescence propagation characteristics of SRSO waveguides with PCs etched into them. A $30 \mathrm{~mW}$ beam from an argon ion laser $(\lambda=514 \mathrm{~nm})$ was focused into a spot of approximately $20 \mu \mathrm{m}$ diameter and used to photoexcite the SRSO. The resulting PL was collected at the edge of the waveguide and collimated using a $90 \times$ microscope objective. This light was then linearly polarized to allow independent detection of both the TE and TM waveguide modes and then coupled into a spectrometer. A nitrogen-cooled $\mathrm{Si}$ CCD detector gave a spectral resolution of $0.1 \mathrm{~nm}$ up to the cut off wavelength of $1.1 \mu \mathrm{m}$. To measure the effect of the PC on the waveguided $\mathrm{PL}$, a ratio of intensities as a function of wavelength is taken to compare the emission for different positions of the excitation spot relative to the PC (shown schematically in the inset of Fig. 2).

The effect of the PC on the luminescence of the SRSO is directly seen by comparing the PL transmitted from an excitation spot $20 \mu \mathrm{m}$ before the near side of the PC [Fig. 2(b)], with the spectrum which has passed through the PC from an excitation spot $20 \mu \mathrm{m}$ beyond the far side [Fig. 2(c)]. This waveguided PL is referenced to that measured from an excitation spot at the edge of the waveguide [Fig. 2(a)], which is thus unaffected by waveguide absorption. The additional broad peak in (b) from $850-900 \mathrm{~nm}$ is a result of light with a wavelength lying within the photonic bandgap (PBG) being efficiently reflected back from the PC, thus increasing the collected PL intensity. In contrast, the dip in (c) over the same wavelength range arises from light within the $P B G$ being rejected by the PC. The contributive effects of the PC reflection to the observed bandgap make this a favorable technique for perceiving weaker bandgap features.

Measurements for 20-row PCs with $610 \mathrm{~nm}$ lattice pitch were carried out for hole diameters of 240, 250, and $280 \mathrm{~nm}$. Downloaded 04 Mar 2005 to 152.78.67.79. Redistribution subject

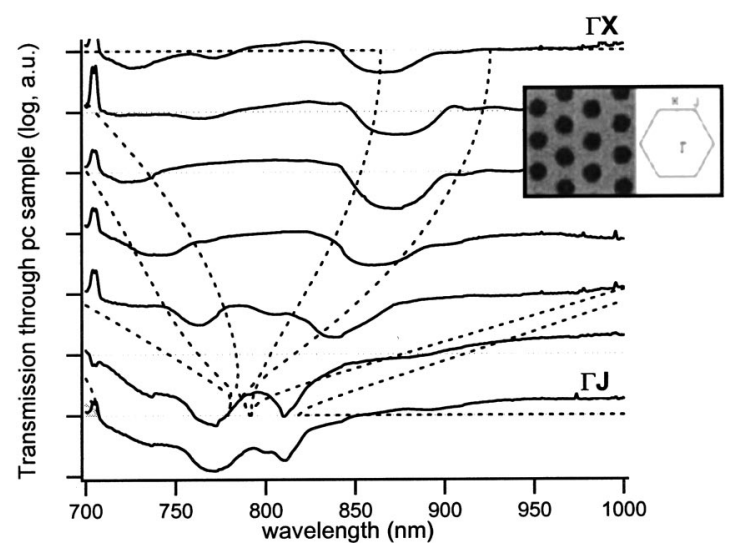

FIG. 3. Experimental TE transmission spectra (solid lines) showing PBGs in waveguides of SRSO. The regions enclosed by dashed lines delineate the theoretically expected location of bandgaps at the corresponding directions of propagation, found using a $2 \mathrm{D}$ plane wave expansion technique [see also Fig. 4(a)]. The inset gives propagation directions $\Gamma X$ and $\Gamma J$.

The filling fractions of these PCs are $0.12,0.13$, and 0.17 respectively. PCs were fabricated with different orientations to the incident light, from the $\Gamma X$ to the $\Gamma J$ direction in $5^{\circ}$ steps. This range covers all angles in the reduced Brillouin zone (BZ) (see inset, Fig. 3). The wavelength ranges of the PBGs can be deduced from the ratio of the PL intensity passing through the PC to that which has not, as shown in Fig. 3. Extinction values as high as one order of magnitude were observed, as shown here for bandgap features in the TE polarization. Different features are seen in the TM polarization, which is consistent with the simulations described later in this letter.

Band diagrams generated using a 2D plane wave technique $^{14}$ are shown in Fig. 4(a). This expansion was carried out for a triangular lattice PC with a pitch of $610 \mathrm{~nm}$ and a hole diameter of $240 \mathrm{~nm}$ in a dielectric material with the same refractive index as that of the fabricated SRSO (measured by ellipsometry). The results of the plane wave expansion were unfolded at different propagation directions giving shaded bandgap regions overlayed on the data of Fig. 3. These results have been shifted $65 \mathrm{~nm}$ towards shorter wavelengths to give a good overlap with the experimental results (as expected from the shortcomings of a 2D model in a threedimensional (3D) waveguide, but in line with previous corroborations between $2 \mathrm{D}$ and $3 \mathrm{D}$ theories).

Several PCs were fabricated at the same pitch size and diameter, and were found to exhibit similar photonic properties, demonstrating the reliability and reproducibility of the process. Samples were also fabricated with 40 and 60 rows producing bandgaps at the same wavelengths, but with broader features from increased scattering within the extra rows. From this data, we find, as expected, that as the filling fraction of the PC increases, the PBGs of that PC shift towards the higher energy wavelengths.

Finite-difference time domain (FDTD) simulations ${ }^{15}$ were also carried out for the same PC sample using 500 rows though with a core refractive index of $n=1.7$. Comparable results are shown as dashed lines in Fig. 4(b), with experimental results on the same diagram (solid lines). These simulations were carried out for the same propagation directions through the crystal as the experiment, and the corresponding AIP license or copyright, see http://apl.aip.org/apl/copyright.jsp 

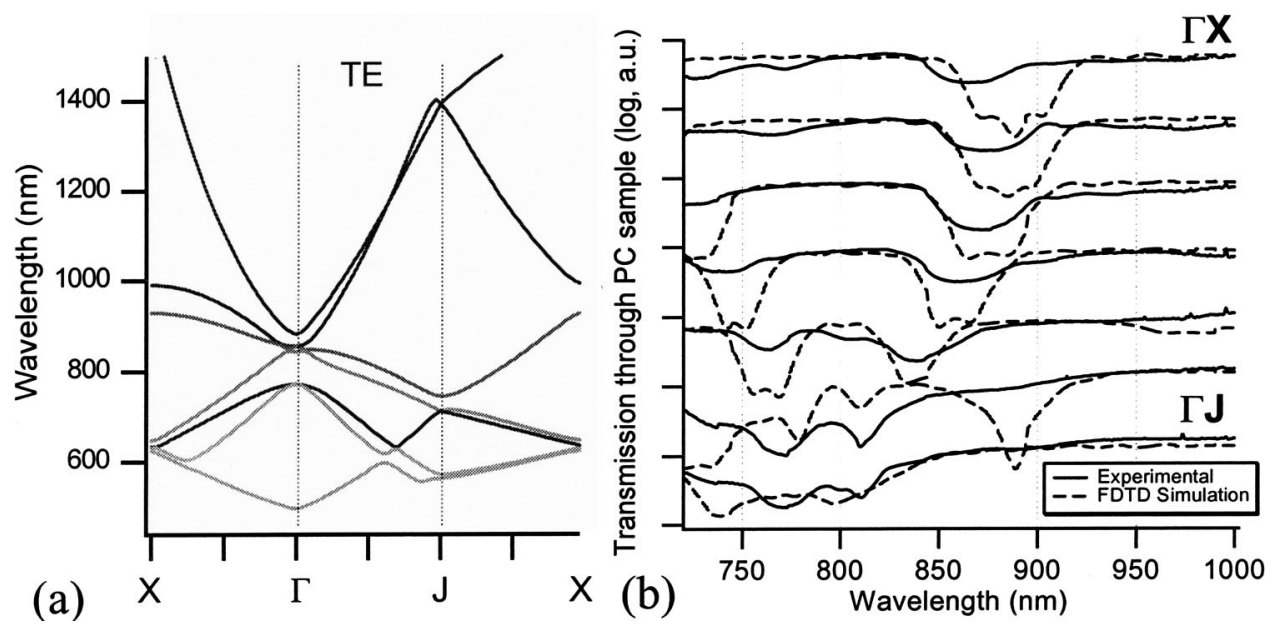

FIG. 4. (a) Band diagrams generated using a standard 2D plane wave expansion technique. (b) Experimental results (solid lines) and FDTD simulation results (dashed lines) showing PBGs in waveguides of SRSO as the direction of propagation is rotated between the $\Gamma J$ and $\Gamma X$ directions (see inset Fig. 3). The curves on a log scale have been offset for clarity.

pairs have been placed together. It should be noted that the FDTD simulation results were again shifted $30 \mathrm{~nm}$ toward shorter wavelengths to overlap with the experimental results. This shift in wavelength is unsurprising, being of the scale and in the direction to compensate for the measured value for refractive index of $n=1.685$.

Figures 3 and 4 illustrate the good correspondence between experimental results and theory, gained by very different methods. In both diagrams, a clear pair of PBGs (between third and fourth, and fourth and fifth bands) may be seen which converge as the direction of propagation rotates from $\Gamma X$ towards $\Gamma J$. For instance, the bandgaps centered at 855 and $750 \mathrm{~nm}$ at $15^{\circ}$ are clearly evident in both theory and experiment. As these bandgaps merge (along $\Gamma J$ ) to form a multifeatured gap, higher and lower bandgaps also converge at this degeneracy point. Features around $\Gamma X$ not represented by the FDTD simulation may arise from waveguide transmission features, while the strength of the modes and their steepness is reduced, probably by angular averaging from scattering of the emission within the PC. The only FDTDsimulated feature that does not show up clearly in the experimental results is the $900 \mathrm{~nm}$ feature for $5^{\circ}$. This is attributed to the low number of rows used in the measured samples relative to the FDTD simulation.

The flexibility and reliability of the PC fabrication process described, together with the good agreement with theory, suggests significant potential for further bandgap engineering in our SRSO waveguides. In particular, the PBG demonstrated in this letter do not include the primary bandgap, which is situated around $1500 \mathrm{~nm}$ for PCs of this lattice pitch size. The PBGs discussed here are higher order bandgaps, which we would anticipate have less broad features and much lower extinction coefficients than for the primary bandgap from theory. ${ }^{16}$ Work currently in progress includes the manufacture of PCs with smaller lattice pitch in order to shift the primary bandgap into the wavelength range of interest for undoped SRSO. In the longer term, the use of 2D PCs and, possibly, engineered combinations of onedimensional and 2D photonic structures, has the potential to allow highly elaborate tailoring of the propagation and group-velocity dispersion of the core PL through the wave- guide structure. One foreseeable application, for example, would be the incorporation of PC "superprisms" 17 into the active region in order to separate different wavelength components of the in-plane luminescence into different directions within a small area of the waveguide. We believe these possibilities have very exciting implications for the development of integrated PC-based optical circuits.

In summary, waveguides of photoluminescent SRSO were patterned with 2D triangular photonic crystals, and the PL of the core material was observed to be significantly modified by the higher-order PBGs of the structure. FDTD simulation and 2D plane wave expansion matched closely the data and can thus be used to explain the results satisfactorily. This work opens the way towards integrated PC-based circuitry within CMOS-compatible silicon microelectronics.

${ }^{1}$ J. R. Haynes and H. B. Briggs, Phys. Rev. 86, 647 (1952).

${ }^{2}$ L. T. Canham, Appl. Phys. Lett. 57, 1046 (1990).

${ }^{3}$ V. Vinciguerra, G. Franzò, F. Priolo, F. Iacona, and C. Spinella, J. Appl. Phys. 87, 8165 (2000).

${ }^{4}$ F. Iacona, G. Franzò, and C. Spinella, J. Appl. Phys. 87, 1295 (2000).

${ }^{5}$ E. Yablonovitch, Phys. Rev. Lett. 58, 2059 (1987).

${ }^{6}$ M. E. Zoorob, M. D. B. Charlton, G. J. Parker, J. J. Baumberg, and M. C. Netti, Nature (London) 404, 740 (2000).

${ }^{7}$ H. Kosaka, T. Kawashima, A. Tomita, M. Notomi, T. Tamamura, T. Sato, and S. Kawakami, J. Lightwave Technol. 17, 2032 (1999).

${ }^{8}$ P. Ferrand, D. Loi, and R. Romestain, Appl. Phys. Lett. 79, 3017 (2001).

${ }^{9}$ A. Talneau, L. Le Gouezigou, N. Bouadma, M. Kafesaki, and C. M. Soukoulis, Appl. Phys. Lett. 80, 547 (2002).

${ }^{10}$ M. C. Netti, C. E. Finlayson, J. J. Baumberg, M. D. B. Charlton, M. E. Zoorob, J. S. Wilkinson, and G. J. Parker, Appl. Phys. Lett. 81, 3927 (2002).

${ }^{11}$ M. Boroditsky, T. F. Krauss, R. Coccioli, R. Vrijen, and R. Bhat, Appl. Phys. Lett. 75, 1036 (1999).

${ }^{12}$ U. Grüning, V. Lehmann, and C. M. Engelhardt, Appl. Phys. Lett. 66, 3254 (1995).

${ }^{13}$ R. T. Neal, M. D. C. Charlton, G. J. Parker, C. E. Finlayson, M. C. Netti, and J. J. Baumberg, Appl. Phys. Lett. 83, 4598 (2003).

${ }^{14}$ K. Sakoda, Optical Properties of Photonic Crystals (Springer, Heidelberg, Germany, 2001).

${ }^{15}$ A. Taflove, Advances in Computational Electrodynamics; The FiniteDifference Time-Domain Method (Artech House, Norwood, 1998).

${ }^{16}$ J. D. Joannopoulos, R. D. Meade, and J. N. Winn, Photonic Crystals (Princeton University Press, Princeton, NJ, 1995).

${ }^{17}$ H. Kosaka, T. Kawashima, A. Tomita, T. Tamamura, T. Sato, and S. Kawakami, Phys. Rev. B 58, 10096 (1998). 